\title{
3D Hologram in Futuristic Classroom: A Review
}

\author{
Chandra Reka Ramachandiran ${ }^{1}$, Mien May Chong ${ }^{1}$, Preethi Subramanian ${ }^{1}$ \\ ${ }^{1}$ School of Computing, Asia Pacific University, Technology Park Malaysia, 57000 Kuala Lumpur, Kuala Lumpur, Malaysia
}

\section{Article Info}

Received Dec 22, 2018

\section{Keyword:}

Education

Futuristic Classroom

Holography

3D Hologram

\begin{abstract}
Holography is a photographic method that records the light dispersed from a body and then produces a realistic image identified as three-dimensional Holograms. The hologram reflects and transmits using a point source of incandescent light or two-channel transmission hologram. In the world of education, 3D holograms can be integrated into mixed reality classrooms to ease the teaching and learning process. The significance of this application is to enable the 21 st Century learners to experience the realistic content via 3D Holograms, thereby improving their learning curves. This paper highlights the fundamental concepts of hologram and discusses the diversified implementations of hologram as a mixed reality simulator in the futuristic classrooms. Recent technological advancements in the field of education and evidence from successful implementation of the mixed reality approach in educational research has also been debated. However, the application of Holograms in education is still confined within boundaries.
\end{abstract}

\section{Corresponding Author:}

Chandra Reka Ramachandiran,

School of Computing,

Asia Pacific University,

Technology Park Malaysia,

57000 Kuala Lumpur, Kuala Lumpur, Malaysia,

Email: chandra.reka@apu.edu.my

\section{Introduction}

In the 21 st century learning, classroom demonstration is still one of the effective ways to capture the attention of the students in the teaching and learning process. Most of the class demonstrations require long hours of preparation time before the actual teaching occurs. Traditional teaching and learning process is carried out either in the classrooms or appropriate laboratory environments. For instance, if an engineering lecturer wants to explain about the internal structure of an internal combustion engine to the students, it is highly difficult to haul the engine to the classroom and lift the engine up in the class in order to elaborate about the internal parts of the engine. In order to overcome this hurdle in teaching, 3D scanning had been employed in the field of nursing as highlighted in [1]. In this study, the cardiovascular structure of heart was developed using the 3D scanning method. The image was converted into a 3D vector layer object and then segmented into different images with different viewing angles to produce a 3D floating image. This image was projected using a selfmade holographic projection display equipment. The researchers found that implementation of the 3D Hologram of the floating heart enhanced the on-site teaching and learning experience of the instructors as well as the learners [1]. Thus, issues related to content delivery can be rectified using recent technology. The 3D hologram using the mixed reality approach is an exemplary example to solve the mentioned issue. Figure 1 
depicts the architecture of 3D Holograms to explore the 'anatomy' of a virtual car in a classroom via the Microsoft HoloLens [2].

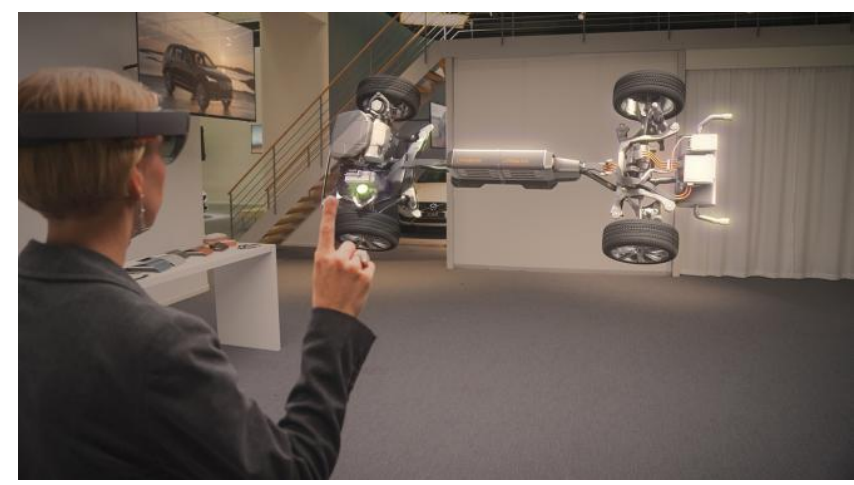

Figure 1. Classroom Demonstration using Microsoft HoloLens

3D Hologram is one of the most advanced technology which could benefit several specializations in the field of education. It is a technology that aims in assisting the students and teachers to view devices that cannot be available in a traditional teaching and learning environment. Distance learning and online learning approach has also gained positive reviews by using this technology. The 3D Hologram works by creating a threedimensional image illusion. In order to produce the illusions, firstly, a source of light is focused on the surface of an item and the light gets dispersed. Meanwhile, a second source of light is employed to radiate the same item and thereby create an interference between both the sources of light. This results in a connection between both the light sources thereby causing a diffraction that gives the impression similar to a 3D image [3].

Furthermore, the 3D Holograms also provide a learning platform that enables the education institutions to offer a Virtual Teacher who is physically located at a different state or country and still able to deliver their lessons. This technology is beyond the video conferencing approach since the 3D hologram facilitates the virtual image of the educator and it seems as if the person is really in the premise and seamless interaction can occur with the students [3-4]. This is an emerging trend in technology that can create multilevel interactions that keeps the students and educators connected regardless of their physical location. Figure 2 shows a sample of the applied technology via the CNN Hologram Architecture [5].

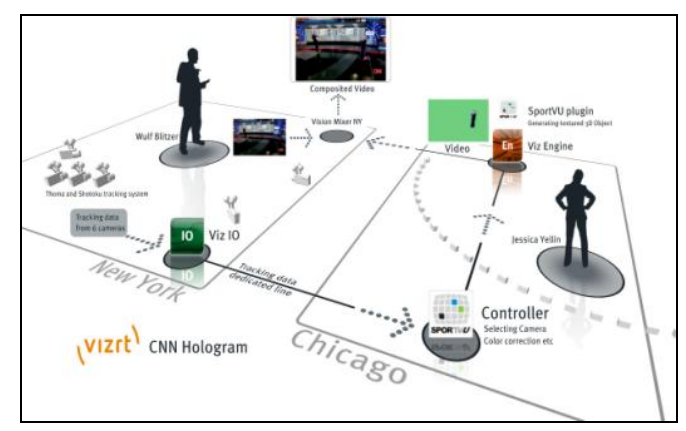

Figure 2: CNN Hologram Architecture

In addition to bringing the educator on live using the 3DHolograms, it can also bring things such as characters and archaeological findings to be on live. Researchers also found that 3D holograms enhance oral history's claim to truth and historical indexicality in a way that 2D cannot achieve [1]. This will allow the students to learn visually and have a better understanding about the subject matter. Specifically, holograms in engineering classrooms will aid the student to have an enhanced understanding of the $3 \mathrm{D}$ models that is being built. The students will be able to gauge the success and outcome of the developed model before the construction of the real model. Hence, it is cost efficient and the design factors are identified at an early stage to ensure higher success rate. For instance, a 3D Hologram classroom was deployed in a remote location in Nigeria to conduct lessons for the students. It was a proposed solution to address the manpower issue in the country $[2,6]$. However, it is crucial to understand that the 3D Hologram technology can only be implemented with good 
infrastructure facility such as reliable bandwidth internet connection to interpret the image of the 3D Hologram.

\section{Hologram Technology}

Holograms have been existing at least for the last two decades. The first person to invent this hologram is Dennis Gabor. Holograms look easy to implement, but in the real world, it is different. The implementation is much more complicated and is elaborated in the following sections.

\subsection{The Science of Holography}

Holography is a term that was derived from the Greek language, where the word 'holo' means a whole, and the word 'graphy' means a record. Thus, the word 'holography' is defined as a whole record. Holography can record all the information about an object in the form of an image. This is similar to audio records where music is created by musical instruments or vocal cords. The music thus produced is encoded and recorded using suitable devices so that it can be played at a future time without the presence of the original instrument [7]. By exploring this image, the user can get all the object's information from it as elaborated in [7-9]. Figure 3 shows the theoretical process of the creation of the hologram discussed in [7].

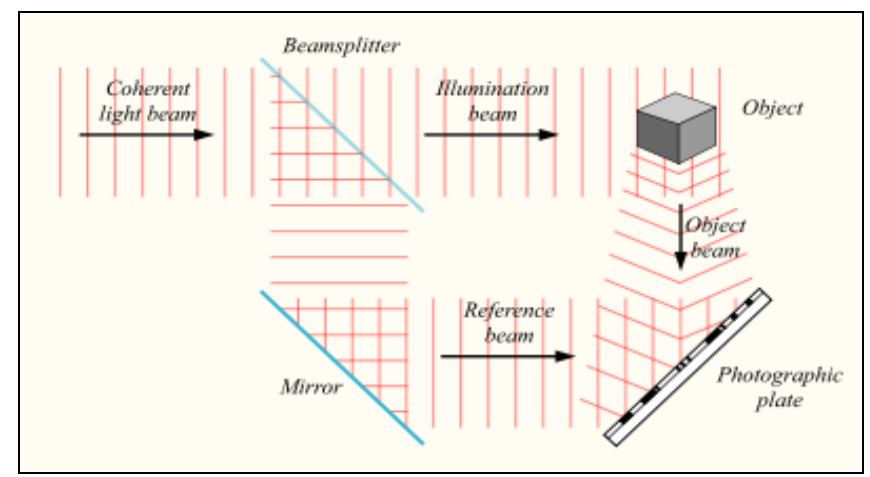

Figure 3. Hologram Recording

\subsection{Classification of Holograms}

Initially, the holograms that were produced were flat two-dimensional transparencies that were obtained from the appropriate considerably coherent light of a sodium vapour lamp. The three-dimensional holograms originated in 1962 with the experiments conducted at the University of Michigan by Emmett Leith and Juris Upatienks as mentioned in [1]. The holograms were used in the field of optical engineering that became popular after the World War II. This evolution of holograms continued till today. According to [9], the hologram has been improved to such an extent that it is possible to project a comprehensive 360-degree holographic display. Currently, there are many types of hologram which have been invented by the researchers. In this sub-section, the major types of the hologram are discussed.

\section{i. Transmission Hologram}

Transmission hologram mainly depends on laser light and it gets produced when the object and reference beams are incident on the recording medium from the same side. Figure 4 depicts the findings from [8] can be enhanced to add several mirrors to deviate the beams in the required directions to produce holograms. 


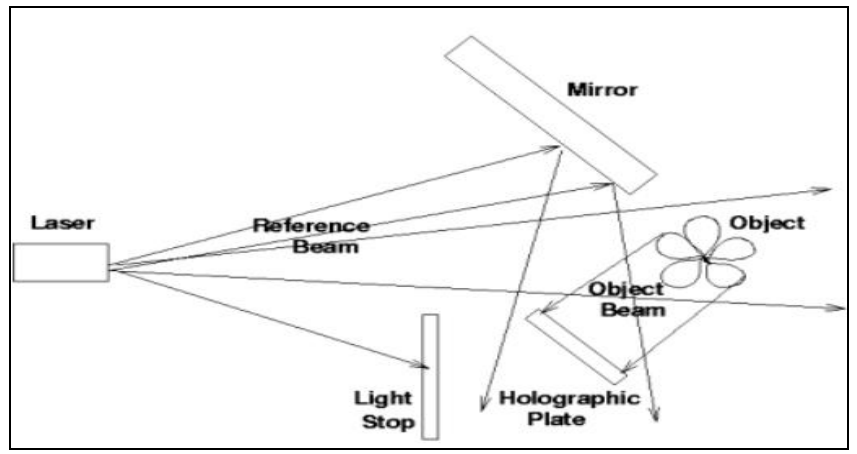

Figure 4. Process to produce the Transmission Hologram

\section{ii. Reflection Hologram}

The basic concept of reflection employs a white light from sources such as spot light, flash light and so on. When two beams from opposite directions approach the holographic plate, reflection happens. The reflected image thus produced is stored in a thick emulsion and can be viewed in white light [8]. The illustration process of the reflection hologram is shown in Figure 5.

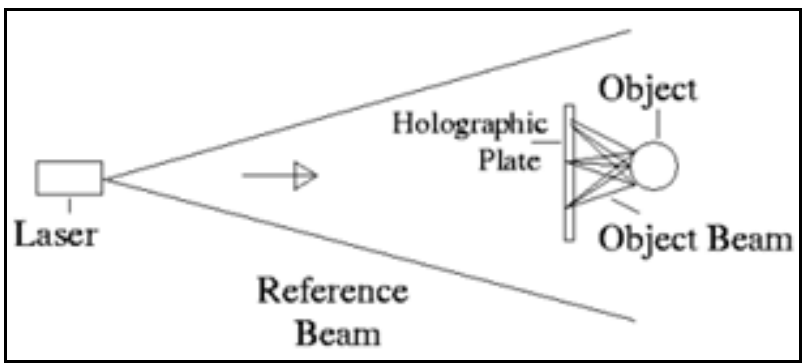

Figure 5. Hologram Reflection Process

iii. Special glasses type

This type of glass permits the light to pass through and also permits another light to produce images on the glasses. Microsoft produced HoloLens in 2016 [9]. This Hololens is the earliest self-contained, holographic computer that aids the user to engross with the digital content and also work together with the other holograms that exists [10]. Figure 6 displays the special glasses which produces 3D holograms as illustrated in article [8, $10]$.

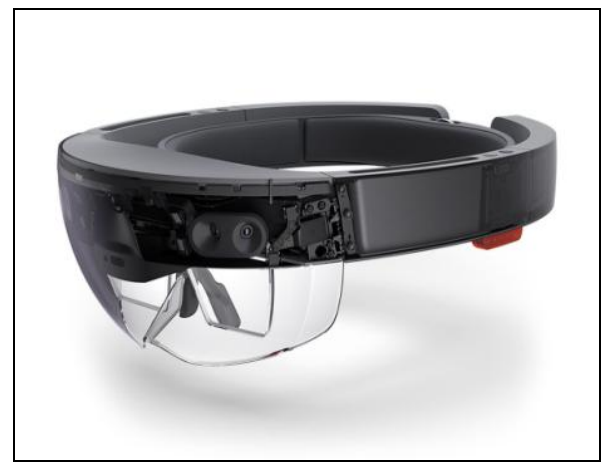

Figure 6. Microsoft Hololens 


\section{3D Hologram as a Simulator in Education}

Nowadays, interactive learning is one of the important pedagogical approaches in the education area. Interactive learning is an applied and pro-active approach to facilitate the learners to improve their involvement in the learning process. Positively, this approach also helps the learners to preserve further information. Hologram technology can be employed in the interactive learning approach to enhance the learning process.

In the medical field, the lecturers can conduct a live simulation for their students to learn a surgery procedure by using 3D Hologram technology as shown in Figure 7 [11].

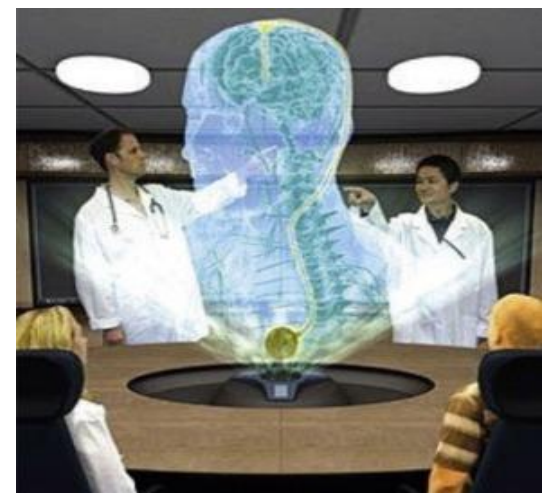

Figure 7. Hologram in Medical Science (Mishra, 2017)

According to [11], there are several hologram systems in the current market that have been developed by the companies for the medical area, such as EchoPixel True 3D, and HoloPatient. EchoPixel True 3D is one of the systems that uses the special lightweight glasses. It allows the users to turn images into 3D holographic visuals. A stylus is employed to travel and communicate with the organs, tissues and vessels that can be viewed on their workspaces in real time; while the HoloPatient is one of a suite of educational applications that developed by Pearson [12-13]. In this application, user have to press a button on the goggles of the Microsoft Hololens, a three dimensional, life-size patient will be appeared in the classroom [13].

For the architectural or engineering teachers, they can use the hologram to display the 3D imaging building or hardware structure to the students. Through this way, students can easily feel and see the structure in real example instead of just look into the 2D image from the book or go to the construction area to see the structure. There are some holograms systems were built for the architecture or engineering area. For instance, Zebra Imaging System is one of the current famous system that used hologram technique in the architecture or engineering domain of study [14].

\section{Challenges of Hologram Implementation}

There are some limitations faced in the practical applications of the hologram. Aspect restrictions have been discussed in this section.

\subsection{Light Modulation}

Holograms can generally be observed from few directions only in order to capture the details of the image. The holographic enactment of rapper Tupac Shakur at Coachella in 2012 threw the world into a state of frenzy. Gigantic machines and glasses were placed surrounding the platform in that concert and also in the programme by Michael Jackson in 2014. The holograms produced were so captivating that it tied the viewers to remain in front of the hologram which was projected on the stage. The light modulation limits the directions from which the holograms can be observed similar to the concepts highlighted in [8]. However, a few organizations that are functioning on holograms for cell phones have managed to increase the number of directions to view the hologram but it has been achieved merely at a small scale. 


\subsection{Information encoding}

The Contemporary holographic outcrops like the Tupac's performance are pre-programmed which means that the action is not spontaneous and the entire projection is already imagined and carefully constructed. In such a type of hologram, no action can be modified as it happens. The construction of interactive holograms confuses the data encoding process as the programmers need to predict all probable situations and the responses to them. This interactivity would be more complicated compared to developing video games.

\subsection{Connectivity Issue}

The fundamental concept of producing a hologram is to project the virtual form of an object. So, high speed Internet access is required for such projection to happen seamlessly. Higher bandwidth becomes mandatory if the holographic communication has to be performed through any mobile device. It should also be kept in mind that the bandwidth requirement increases proportionately with the increase in the number of consumers.

\subsection{High Implementation Cost}

The cost of producing holograms is still luxurious as the component prices are costly. Although the prices of light modulators and other components have lowered in recent years, the cost of producing holograms at a big scale remains enormous. Producing the Tupac hologram for Coachella could easily cost around $\$ 250,000$. Hologram communication and its effect on society, business and military is still underexplored. Similar to the other technological innovations, this hologram could also be a disruptive innovation is mass production becomes inexpensive.

\section{Conclusion}

The recent advancement in technology have caused the growth of the usage of holograms especially in classrooms [6, 8]. Research has shown that the usage of holograms in classrooms is a futuristic way to improve the learning and teaching experience of students and teachers respectively. Examples of usage holograms in classroom can be that the teachers can teach virtually as discussed in $[6,8,14]$. Another usage is that teachers can project 3-dimensional objects using holograms which will be beneficial for students especially in the medical and engineering sector as they get to have a better understanding of what they are learning. However, some scholars have proved that the usage of holograms in classrooms have their limitations such as the cost of implementation and maintenance. Since holograms is such an advance technology, implementing and maintaining it will not be cheap as the resources are limited depending on the institution or country. Despite it having its limitations, it cannot be denied that the usage of hologram in classrooms will be beneficial for the education institutions. This technology definitely can be improved and in a few years it should be a mainstream technology that can be used in many institutions.

\section{Acknowledgments}

This study is funded by APU Research Development Grant (RDG), Asia Pacific University, Malaysia.

\section{References}

[1] Chang, Y. M., \& Lai, C. L. (2018). "Floating Heart" Application of Holographic 3D Imaging in Nursing Education. International Journal of Nursing Education, 10(4).

[2] Erickson, S. (2015) Microsoft HoloLens and Volvo Cars explore the future of car buying. Available at: https://blogs.windows.com/devices/2015/11/19/microsoft-hololens-and-volvo-cars-explore-the-future-of-car-buying/\#UJH7v2zPFGFL1fFB.97 (Accessed: 9 January 2019).

[3] Ghuloum, H. (2010) '3D Hologram Technology in Learning Environment The Historical Background of ICT in Education', Proceedings of Information Scientce \& IT Education Conference (InSITE), pp. 693-704.

[4] Golden, S. A. (2017). Augmented 3D holograms in higher education, increasing students' learning outcome scores: A mixed methods study (Doctoral dissertation, Keiser University).

[5] Serrao, J. (2008) Holobama: Holograms' greet $2008 . \quad$ Election at:http://www.telepresenceoptions.com/2008/11/holographic_presenters_jessica/ (Accessed: 9 January 2019). 
[6] Ahmad, S. A., Abdullahi, I. M. and Usman, M. (2014) 'General Attitude and Acceptance of Holography in Teaching Among Lecturers in Nigerian Colleges of Education', The IAFOR Journal of Education Technologies \& Education Special Edition.

[7] Hariharan, P. (2002) Basics of holography. Cambridge university press.

[8] Awad, A. H. and Kharbat, F. F. (2018) 'The first design of a smart hologram for teaching', 2018 Advances in Science and Engineering Technology International Conferences, ASET 2018. IEEE, pp. 1-4. doi: 10.1109/ICASET.2018.8376931.

[9] Kerstein, R. (2018) 'Life through a HoloLens', The Bulletin of the Royal College of Surgeons of England, 100(8), p. 333. doi: 10.1308/rcsbull.2018.333.

[10] Microsoft (2019) Mixed reality: Your world is the canvas. Available at: https://www.microsoft.com/en-us/hololens (Accessed: 16 January 2019).

[11] Mishra, S. (2017) 'Hologram the future of medicine - From Star Wars to clinical imaging', Indian Heart Journal, 69(4), pp. 566-567. doi: 10.1016/j.ihj.2017.07.017.

[12] Orenstein, B. W. (2016) Virtual Radiology: New Viewing Platform Creates Interactive Medical Imaging Holograms, Radiology Today. Available at: https://www.radiologytoday.net/archive/rt1216p14.shtml (Accessed: 16 January 2019).

[13] Kamping-Carder, L. (2018) How Holograms Are Helping Medical Training. Available at: https://www.wsj.com/articles/how-holograms-arehelping-medical-training-1530795601 (Accessed: 16 January 2019).

[14] Imaging, Z. (2017) Zebra Imaging Announces Sale of 3D Holographic Print Assets to HoloTech Switzerland AG. Available at: https://www.prnewswire.com/news-releases/zebra-imaging-announces-sale-of-3d-holographic-print-assets-to-holotech-switzerland-ag300525919.html (Accessed: 16 January 2019). 